\title{
Production de lait de vache dans le delta du fleuve Sénégal : une réelle activité de diversification en systèmes irrigués?
}

\author{
Ch. Corniaux ${ }^{1 *}$ J. Le Mercier ${ }^{2}$ A.T. Dia ${ }^{3}$
}

\begin{abstract}
Mots-clés
Lait de vache - Diversification - Rentabilité - Rizière - Culture irriguée Sénégal - Mauritanie.
\end{abstract}

\begin{abstract}
Résumé
En dépit d'un contexte profondément modifié par la mise en place à grande échelle des cultures irriguées, la production laitière s'est développée sur la rive droite (Mauritanie) et s'est maintenue sur la rive gauche (Sénégal) du delta du fleuve Sénégal. Des travaux menés de juillet 1997 à juin 1999 ont permis de mieux évaluer le degré d'interaction entre l'élevage et l'agriculture. Des résultats sont présentés sur l'utilisation des ressources fourragères et sur certains paramètres technico-économiques. La production laitière est apparue comme une véritable activité rentable de diversification à l'échelle de l'exploitation pour les agropasteurs, relativement nombreux sur la rive gauche. Sur la rive droite, elle a semblé davantage correspondre à une spécialisation. L'avenir de cette activité semble prometteur dans cette zone vouée à devenir un bassin de production laitière pour l'approvisionnement des capitales, Nouakchott et Dakar. Le rôle central du maillon de la collecte a été souligné.
\end{abstract}

\section{INTRODUCTION}

Le delta du fleuve Sénégal était autrefois une région dévolue quasi exclusivement à l'élevage extensif (2). Ce dernier, pratiqué par les Peuls et les Maures, exploitait en saison sèche les abondants parcours de décrue libérés par le retrait des eaux du Sénégal. En saison des pluies, étaient utilisés les pâturages dunaires du diéri pourvus à cette période d'un tapis graminéen de qualité.

Depuis une trentaine d'années, ce contexte a été complètement bouleversé. L'aménagement du delta en vue de la culture irriguée a abouti à une diminution sensible des pâturages de décrue, réduits, d'une part, par l'endiguement important du fleuve et, d'autre part, par la conversion à l'agriculture des surfaces disponibles (casiers rizicoles et maraîchage) (9). En outre, les couloirs traditionnels de transhumance et l'accès aux points d'abreuvement ont été notablement modifiés, voire supprimés dans le waalo. Par ailleurs, la sécheresse chronique qui touche la région depuis les années 70 a sensiblement réduit la valeur fourragère des parcours d'hivernage sur le diéri (3).

\footnotetext{
1. Psi-Sénégal/Cirad-emvt, BP 240, Saint-Louis, Sénégal

2. Psi-Sénégal, BP 240, Saint-Louis, Sénégal

3. Cnerv, BP 167, Nouakchott, Mauritanie

* Auteur pour la correspondance
}

Tél. : +221961 1751 ; fax : +2219613462 ; e-mail : christian.corniaux@cirad.fr
Ces mutations du contexte agroclimatique ont contraint les systèmes d'élevage extensifs à modifier leurs cycles de transhumance, notamment par une utilisation accrue des sous-produits agricoles, et à évoluer vers des modèles plus sédentaires et intensifs $(14,15)$. Dans ce contexte favorable à l'essor de l'agriculture, l'élevage s'est maintenu dans le delta du fleuve Sénégal au sein des systèmes irrigués, contrairement à certaines prédictions pessimistes (7). La production laitière s'est même particulièrement développée sur la rive droite depuis le début des années 90, notamment sous l'impulsion de la Laitière de Mauritanie (LM), structure privée de collecte et de transformation du lait frais $(1,6)$. Sur la rive gauche, la filière est restée informelle et basée sur la vente de lait caillé (5).

Peut-on considérer pour autant que la production de lait de vache correspond réellement à une activité de diversification par rapport à la riziculture dans le delta du fleuve Sénégal ? En d'autres termes, comment la production laitière s'intègre-t-elle aux systèmes de production en irrigué et y a-t-elle sa place à l'avenir ? Ces questions restent fondamentales à un moment où les autorités régionales et nationales reconsidèrent leur position face à l'activité de l'élevage, parent pauvre au regard des investissements considérables dans la culture irriguée depuis trente ans, et à la nécessité de trouver des solutions alternatives à la monoculture du riz.

C'est à ces questions que sont apportés des éléments de réponse, sur la base des travaux de l'équipe Elevage du Pôle systèmes irrigués (Psi) menés de juillet 1997 à juin 1999 sur les rives droite et gauche du delta du fleuve Sénégal. 


\section{MATERIEL ET METHODES}

\section{Zone de l'étude}

Les rives droite et gauche du delta du fleuve Sénégal ont constitué la zone de l'étude (figure 1). Le delta comprend deux grands ensembles, le waalo correspondant aux zones inondables près du fleuve, propices au développement de la riziculture, et le diéri correspondant aux zones dunaires non inondables, favorable aux parcours d'hivernage.

\section{Echantillonnage et enquêtes de terrain}

Au Sénégal, un travail initial a été réalisé fin 1997 sur un échantillon de 150 exploitations afin de caractériser les principaux systèmes d'élevage dans le delta à partir de critères structurels (cheptel, surfaces irriguées cultivées en riz ou en maraîchage) (5). Trente d'entre elles, représentatives de leur diversité (trois par type identifié) et réparties sur l'ensemble du delta (figure 1), ont alors été extraites de cette base. Elles ont été enquêtées mensuellement à partir de juin 1998 par le biais de paramètres technico-économiques comme la démographie, la localisation des troupeaux, le type de pâturage utilisé, la production de lait, les chiffres d'affaires du lait et les dépenses en sous-produits agricoles.

En Mauritanie, un premier travail d'analyse des structures d'exploitation a été effectué en décembre 1998 auprès d'un échantillon de 50 producteurs laitiers, fournisseurs à l'usine de collecte de Rosso (figure 1). Afin d'avoir une vision dynamique de la production et de la commercialisation du lait de vache, l'étude précédente a été prolongée par un suivi individuel de ces éleveurs au cours de la saison sèche (janvier à juin 1999). L'objectif a été notamment de préciser la stratégie des éleveurs, durant une période critique pour l'alimentation des animaux, en matière d'orientation de leur production et de vente éventuelle de leur lait (6). Trois passages ont ainsi été réalisés aux mois de février, d'avril et de juin 1999. Le questionnaire a été basé sur le nombre de vaches traites, leur localisation, les quantités de lait produites, la destination du lait et la complémentation alimentaire (quantité, prix).

\section{Traitement des données}

Deux typologies, l'une sur la rive droite, la seconde sur la rive gauche, ont été établies par l'analyse en composantes principales. Les variables actives étaient des variables structurelles (nombre de bovins, surfaces exploitées en riz et en maraîchage), techniques (production de lait et de viande) et économiques (chiffres d'affaires pour le lait, dépenses en sous-produits agricoles).

L'étude du système d'alimentation a été focalisée sur les parcours des vaches laitières (type, localisation, période) et l'usage éventuel de sous-produits agricoles dans leur alimentation (type, période, quantité) de juin 1998 à juin 1999.

Afin de pouvoir comparer les situations économiques des producteurs laitiers des deux rives, les auteurs se sont basés sur les critères communs à leur disposition, le chiffre d'affaires pour le lait et les dépenses en sous-produits agricoles effectués au cours de la saison sèche 1999 (décembre 1998 à juin 1999). Le chiffre d'affaires pour le lait n'a pas pris en compte l'autoconsommation, ni le don, ni la part bue par les veaux. En revanche, le lait vendu sur les circuits informels de la rive droite a été ajouté à celui vendu sur le circuit de collecte de la LM.

\section{RESULTATS}

\section{Typologie}

Sur la rive gauche, de nombreux producteurs laitiers pratiquaient la riziculture et pouvaient être qualifiés d'agro-éleveurs (tableau I). Il s'agissait d'ailleurs essentiellement d'éleveurs Peuls qui s'étaient mis, à des degrés divers, à la riziculture $(9,15)$. Certains éleveurs originaires du diéri (S1) avaient peu accès aux casiers rizicoles. La production laitière ou la production de viande étaient alors leur principale source de revenus (5). Au contraire, chez les agriculteurs Wolofs (S2), la diversification par la production laitière est restée embryonnaire, le nombre et le pourcentage de vaches traites dans le troupeau ayant été très faibles. Elle a semblé plutôt se faire par le maraîchage mais aussi par la production de viande (embouche ovine).

\section{Tableau I}

Principales caractéristiques des producteurs de lait dans le delta (juillet 1998 à juin 1999)

\begin{tabular}{ccccc}
$\begin{array}{c}\text { Nb. } \\
\text { d'éleveurs }\end{array}$ & $\begin{array}{c}\text { Vaches traites } \\
\text { dans le troupeau } \\
(\%)\end{array}$ & $\begin{array}{c}\text { Nb. de vaches } \\
\text { en production }\end{array}$ & $\begin{array}{c}\text { Surface irriguée } \\
\text { exploitée } \\
(\text { ha) }\end{array}$ & $\begin{array}{c}\text { Surface en } \\
\text { maraîchage } \\
\text { (ha) }\end{array}$ \\
\cline { 3 - 5 } & & Moyenne (ET*) & Moyenne (ET) & Moyenne (ET)
\end{tabular}

\section{Groupes sur la rive gauche (Sénégal)}

S1 : éleveurs Peuls du diéri

S2 : agriculteurs-éleveurs Wolofs

S3 : agro-éleveurs Peuls de Ross-Béthio

S4 : riziculteurs-éleveurs Peuls du waalo

S5 : autres **

\section{Groupes sur la rive droite (Mauritanie)}

M1 : éleveurs intensifiés du waalo

M2 : gros éleveurs Maures des dunes

M3 : éleveurs Peuls

M4 : riziculteurs-éleveurs Maures du waalo

M5 : très gros éleveurs Maures des dunes

* Ecart-type

** Eleveurs ne produisant (4 Wolofs) ou ne vendant pas de lait (2 Maures) 


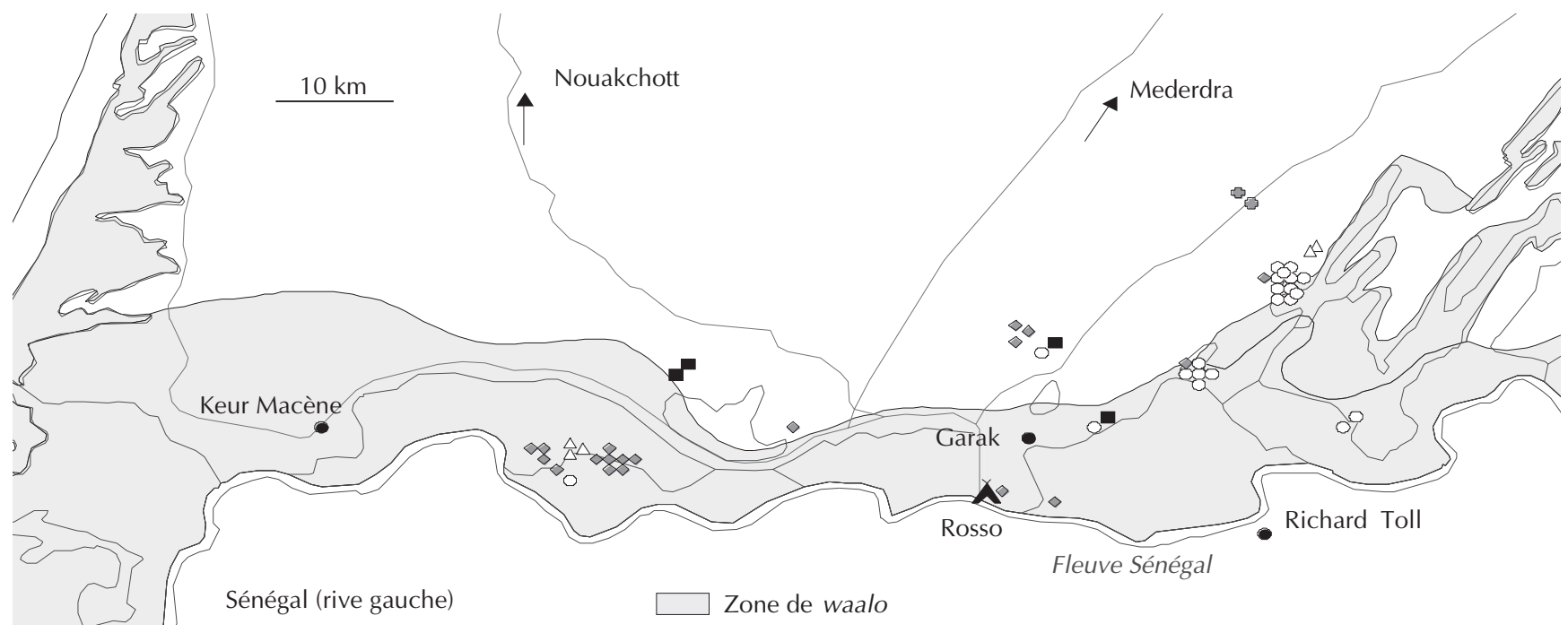

$\checkmark \mathrm{M} 1$ : éleveurs intensifiés du waalo (19)

M2 : gros éleveurs Maures des Dunes (4)

M3 : éleveurs Peuls (19) $\triangle$ M4 : riziculteurs-éleveurs Maures du waalo (5)

5 M5 : très gros éleveurs Maures des Dunes (2)

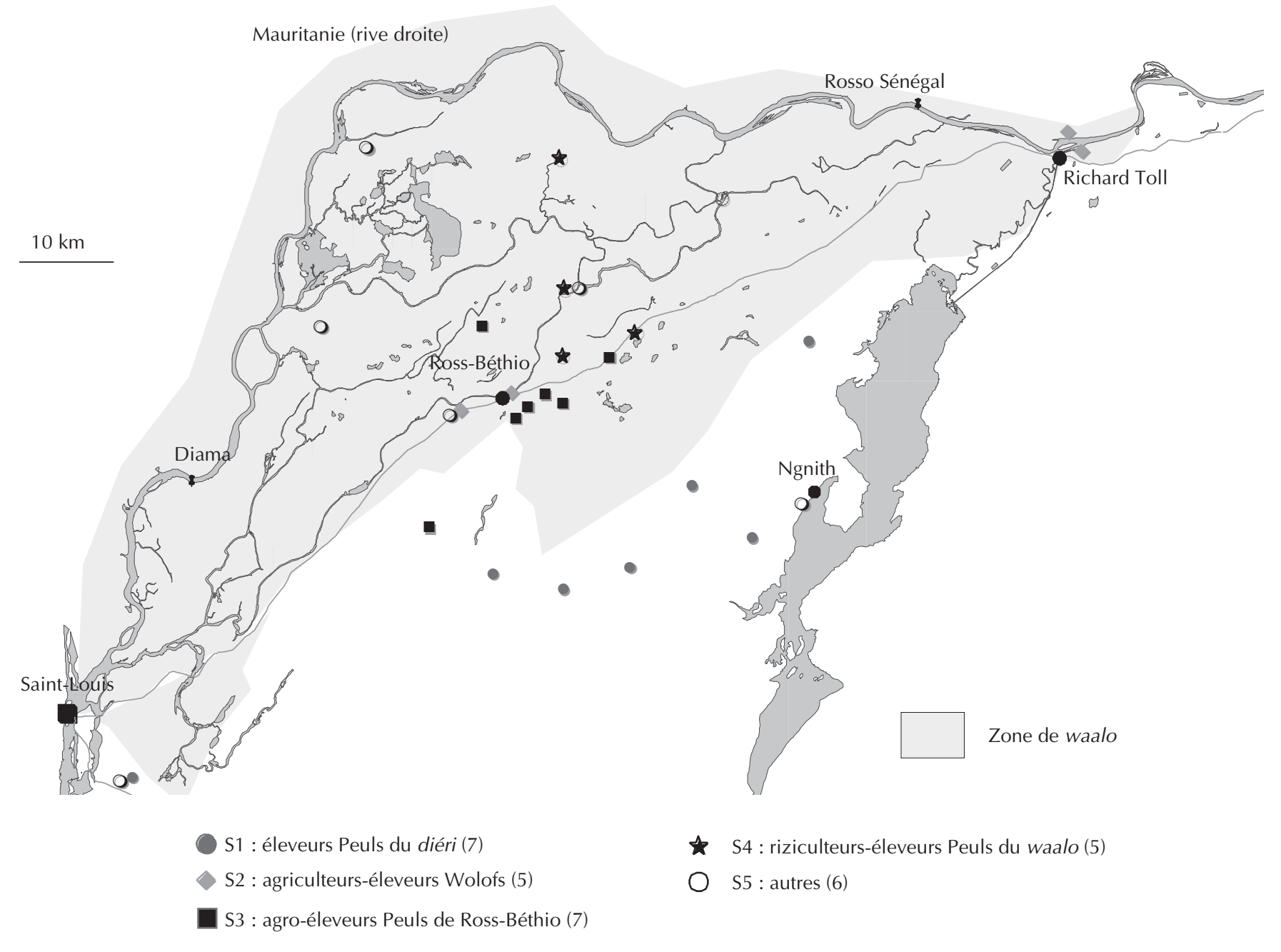

Figure 1 : groupes de producteurs laitiers sur les rives droite et gauche du fleuve Sénégal. 
En Mauritanie, la production laitière correspondait plus souvent à une spécialisation. Cela a été le cas de la plupart des éleveurs fournisseurs de lait à la LM, base de l'échantillon de cette étude sur la rive droite (tableau I), à l'exception notable des agro-éleveurs Maures du waalo (M4). Comme au Sénégal, le maraîchage était peu pratiqué par ces acteurs.

\section{Diagnostic du système d'alimentation : utilisation de l'espace et des ressources fourragères}

\section{Parcours naturels et postculturaux}

Indépendamment de la rive considérée, tous les éleveurs du delta ont envoyé leurs animaux vers les parcours dunaires en saison des pluies et en posthivernage. La quantité et la qualité des fourrages fournis par ces pâturages naturels ont permis la production de lait. Mais à partir du mois de janvier, cette ressource s'est épuisée. Les stratégies des éleveurs ont différé à partir de ce moment-là.

Sur la rive droite, les auteurs ont disposé de peu de mesures permettant de préciser l'utilisation des ressources fourragères du delta par les bovins en saison sèche. Les vaches laitières en production sont cependant restées en majorité à proximité des campements et ont été complémentées à l'auge. Les parcours postculturaux ont par ailleurs été de plus en plus souvent loués par les agriculteurs aux éleveurs. Les gros troupeaux sont partis généralement en transhumance, en dehors du delta.

En revanche, sur la rive gauche, peu d'éleveurs sont partis en transhumance en dehors du delta, même après une année particulièrement sèche (6). Les éleveurs ont trouvé dans le waalo les ressources alimentaires nécessaires au moins à la survie de leurs troupeaux. A l'exception des parcours de décrue du parc du Djoudj et de l'ouest du lac de Guiers, ces ressources ont été issues directement (parcours postculturaux et pré-irrigation des casiers) ou indirectement (abords des canaux) de l'agriculture irriguée.

Cependant, tous les éleveurs de la rive gauche n'ont pas eu les mêmes possibilités d'accès aux parcours postculturaux. Cet accès, généralement gratuit, a été facilité pour les agro-éleveurs (S3, S4). Ils ont laissé pâturer leurs animaux de janvier à juillet dans les casiers ou leurs alentours après la récolte de riz ou en pré-irrigation. En revanche, cet accès a été très limité pour les éleveurs du diéri (S1) qui se sont tournés davantage vers les parcours de décrue afin d'éviter des conflits éventuels avec les agriculteurs $(5,14)$. Sur la rive gauche, la complémentarité des ressources fourragères du diéri et du waalo a ainsi paru indéniable, dans le temps comme dans l'espace.

\section{Sous-produits agricoles}

L'utilisation de sous-produits agricoles et agro-industriels était par ailleurs une pratique courante dans le delta du fleuve Sénégal. Ces sous-produits ont été variés : paille de riz, sons industriel et artisanal de riz, tourteaux d'arachide, mélasse de canne à sucre, fanes et pailles diverses, drêches de tomates (15). A l'exception du tourteau d'arachide, ils sont tous provenus de la zone.

Pour la production laitière, la stratégie des éleveurs du delta a toutefois été différente d'une rive à l'autre. Ainsi, sur la rive droite, les éleveurs (fournisseurs de la LM) ont pratiqué une complémentation alimentaire de production durant toute la saison sèche afin de pallier la faible valeur nutritive des pâturages naturels. Ils ont ainsi maintenu la productivité de leurs vaches laitières à des degrés divers, selon les groupes considérés, et ont même pu l'augmenter en fin de saison lorsque la demande s'est accrue sur le marché de Nouakchott (6). Ils se sont appuyés, bien entendu, sur une filière structurée qui leur a permis d'assurer leur débouché commercial. En revanche, dans le cadre d'une filière informelle, cette pratique a été rare sur la rive gauche. Elle s'est éventuellement rencontrée chez quelques agro-éleveurs ou chez les agriculteurs Wolofs (vaches en stabulation). Pour la grande majorité, il s'agissait d'une complémentation alimentaire de sauvegarde pratiquée en fin de saison sèche (figure 2).

\section{Chiffre d'affaires du lait}

Le chiffre d'affaires n'a tenu compte que du lait vendu (frais ou caillé). Le lait autoconsommé, part élevée de la production au Sénégal (supérieure à 40 p. 100 en moyenne selon Corniaux et coll. ; 5) n'a pas été comptabilisé ici. La part autoconsommée chez les éleveurs mauritaniens est en revanche très faible, inférieure à 10 p. 100 de la production (6) ; la quantité de lait produite a été en effet sensiblement supérieure à celle de la rive gauche alors que la taille des familles a été comparable. Le circuit de collecte rodé et le débouché de vente solvable de la LM ont aussi incité les éleveurs à vendre le lait plutôt qu'à le boire. Le contrôle progressif de la vente du lait par les hommes, au détriment des femmes, risque d'amplifier ce phénomène.

En Mauritanie, le prix d'achat à l'éleveur du litre de lait de vache est fixé à 110 ouguiyas (UM), soit environ 275 Fcfa toute l'année (prix de la LM au cours de cette étude). En revanche, le prix du lait vendu en dehors de ce circuit s'est négocié de $125 \mathrm{Fcfa} / \mathrm{l}$ en saison froide à $500 \mathrm{Fcfa} / \mathrm{l}$ en saison chaude. Sur la rive gauche, le prix du litre de lait caillé ou cru a varié relativement peu tout au long de l'année, de 200 à $350 \mathrm{Fcfa} / \mathrm{l}$. La substitution s'est faite en effet progressivement entre le lait caillé fabriqué à partir du lait frais et
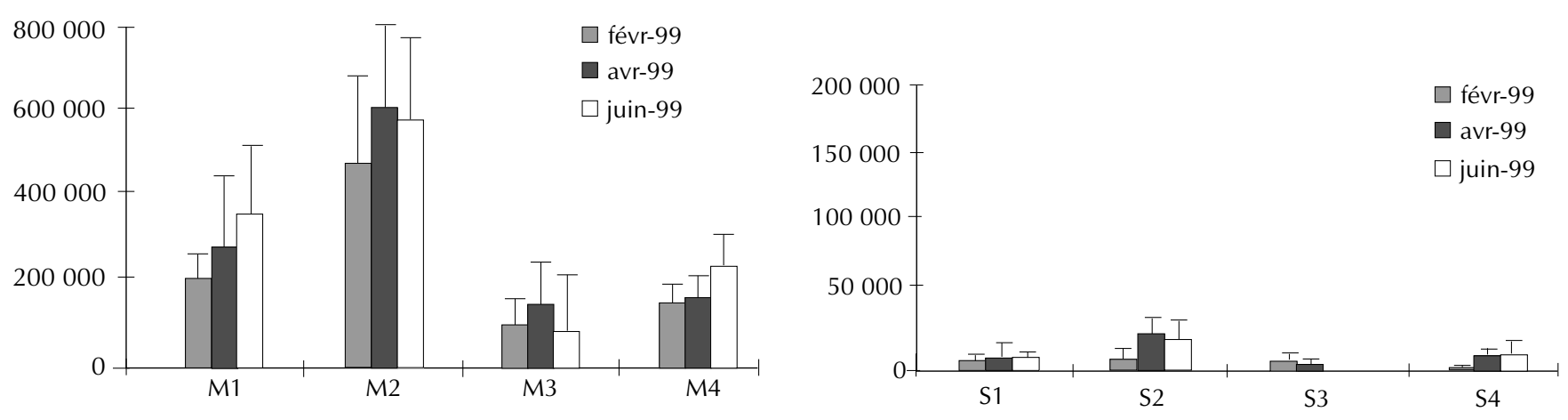

Figure 2 : dépenses mensuelles (FCfa) en sous-produits agricoles et agro-industriels des producteurs laitiers, pendant la saison sèche de 1999, dans le delta du fleuve Sénégal, sur la rive droite en Mauritanie (M), sur la rive gauche au Sénégal (S). M5 = 2,4, 2,8, 2,7 millions de Fcfa respectivement pour février, avril et juin 1999. 
celui fabriqué à partir du lait en poudre importé (soit environ 250 Fcfa le litre reconstitué).

Le chiffre d'affaires pour le lait a permis d'apprécier le flux réel lié à l'activité commerciale de ce produit. La figure 3 montre qu'il a été éminemment variable entre les deux rives, en partie du fait de l'activité commerciale régulière des éleveurs mauritaniens avec la structure privée de collecte. Au Sénégal, à partir du mois de décembre et consécutivement à l'appauvrissement des parcours d'hivernage, les meilleurs chiffres d'affaires mensuels, voire annuels (tableau II), n'ont même pas atteint les chiffres planchers mauritaniens et sont restés en moyenne par groupe inférieurs à 100000 Fcfa par mois. De plus, ce chiffre d'affaires a diminué rapidement à partir du début de la saison sèche et est devenu très faible dès le mois d'avril. Les agropasteurs des groupes S3 et S4, qui ont dépassé les 100000 Fcfa mensuels en octobre et en novembre, sont parvenus à maintenir un chiffre d'affaires minimum en début de saison sèche (utilisation des ressources fourragères des parcours postculturaux ou maintien d'un effectif en production important).

\section{Dépenses en sous-produits agricoles}

Dans le delta, l'essentiel des sous-produits, qui représentait la principale charge consentie par les éleveurs, a été acheté en saison sèche. Sur la rive droite, ces dépenses ont exclusivement été destinées aux vaches laitières en production. Sur la rive gauche, elles ont généralement été réalisées pour la sauvegarde du bétail en fin de saison sèche. Seules quelques vaches laitières généralement élevées en case et des animaux à l'embouche ont reçu une complémentation de production. Dans ce cadre, les dépenses en sous-produits agricoles effectuées par les éleveurs sénégalais ont paru très faibles comparativement à celles des éleveurs mauritaniens (figure 2). Il existait cependant des différences sensibles entre les groupes.
Ces différences ont souvent reflété le nombre d'animaux complémentés, les plus grosses dépenses ayant été consenties pour les plus gros troupeaux. Mais certains éleveurs ont également choisi de réduire sensiblement la complémentation en saison sèche. Il s'agissait notamment des Peuls (M3) qui ne souhaitaient pas risquer leur revenu devant le coût onéreux des sous-produits agricoles. D'autres en Mauritanie ont accepté des dépenses importantes sensiblement égales, voire supérieures, à leur chiffre d'affaires à cette période de l'année, pour préserver l'état corporel de leurs vaches (investissement sur une prochaine lactation rémunératrice en hivernage).

\section{DISCUSSION}

\section{Diversification : à quelle échelle?}

\section{De la parcelle à l'exploitation}

La notion de diversification est souvent interprétée dans le delta du fleuve Sénégal comme étant l'introduction d'une alternative agricole rentable à la monoculture du riz. Elle est ainsi généralement considérée par les agriculteurs et les agronomes à l'échelle de la parcelle : la culture du riz est remplacée par une autre culture plus rentable ou moins dégradante pour l'environnement. Pourtant, dans le delta du fleuve Sénégal, contrairement à d'autres zones irriguées de l'Afrique de l'Ouest ou de la vallée, l'introduction de cultures maraîchères (tomates, oignons, gombo...) ou de rente (arachide, sorgho, maïs...) se fait rarement en rotation avec du riz dans les casiers $(8,13)$. Aussi, la diversification concerne les activités agricoles qui doivent être considérées à l'échelle de l'exploitation.

C'est à ce niveau qu'il faut considérer la place de la production laitière, ou plus globalement de l'activité élevage, dans les systèmes

\section{Tableau II}

Chiffre d'affaires annuel (juillet 1998 à juin 1999) pour le lait des éleveurs sénégalais

$\begin{array}{lcc} & \text { Nb. d'éleveurs } & \text { Chiffre d'affaires (KFcfa) } \\ & & \text { Mnnuel } \\ & & \\ \text { S1 : éleveurs Peuls du diéri } & 8 & 228 \\ \text { S2 : agriculteurs-éleveurs Wolofs } & 5 & 97 \\ \text { S3 : agro-éleveurs Peuls de Ross-Béthio } & 6 & 707 \\ \text { S4 : riziculteurs-éleveurs Peuls du waalo } & 5 & 560 \\ \end{array}$
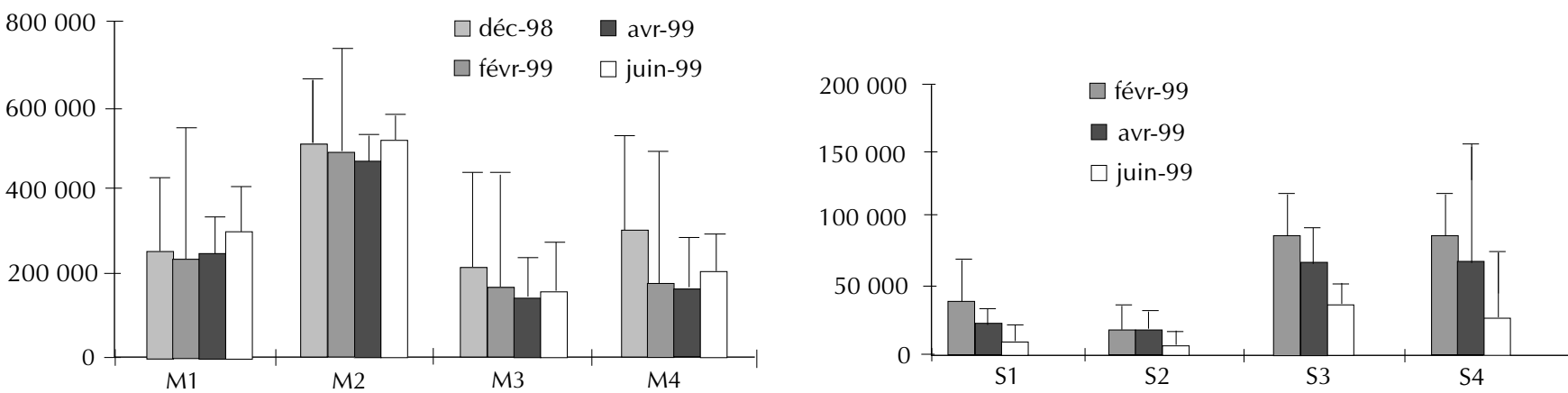

Figure 3 : chiffre d'affaires mensuel (FCfa) pour le lait des producteurs laitiers, pendant la saison sèche de 1999, dans le delta du fleuve Sénégal, sur la rive droite en Mauritanie (M), sur la rive gauche au Sénégal (S). M5 = 5,7, 3,6, 3,7, 3,8 millions de Fcfa respectivement pour les mois de décembre 1998, février, avril et juin 1999. 
irrigués du delta du fleuve Sénégal. En effet, à l'échelle de la parcelle, la traction animale et les cultures fourragères se sont très peu développées à ce jour. L'essor des cultures fourragères devrait toutefois être rapide sur la rive droite dans les années à venir, notamment chez les riziculteurs-éleveurs Maures du waalo (M4). En revanche, comme cela a été noté ci-dessus, à l'échelle de l'exploitation, de nombreux producteurs laitiers pratiquent également la riziculture. C'est en particulier le cas au Sénégal où plusieurs groupes d'agro-éleveurs peuvent être distingués. Sur la rive gauche, il y a donc une réelle diversification des activités agricoles par la production laitière à l'échelle de l'exploitation.

\section{A l'échelle du terroir}

La sédentarisation des éleveurs, liée principalement à la riziculture sur la rive gauche et à la production laitière sur la rive droite, les oblige à trouver sur place les ressources fourragères nécessaires à l'alimentation de leurs troupeaux. Ceci est possible grâce au potentiel issu des cultures irriguées. A l'échelle du delta, il faut par conséquent noter une véritable complémentarité des activités élevage et agriculture, à l'image du partage dans le temps des ressources du diéri et du waalo. C'est parce qu'il a su s'adapter aux systèmes irrigués, à défaut de s'intégrer totalement, que l'élevage, plus particulièrement la production laitière, constitue une activité de diversification à cette échelle.

\section{Rentabilité de la production laitière comparée aux autres productions de diversification}

La production laitière doit être rentable pour se pérenniser et justifier l'engagement d'investissements lourds à destination de l'activité élevage au sein des systèmes irrigués. Des différences existent toutefois entre les deux rives.

Ainsi, en Mauritanie, dans le cadre d'une filière organisée, il s'agit d'une nécessité fondamentalement économique. Les producteurs laitiers cherchent à tirer un revenu optimal de leur activité, quitte à investir dans une alimentation coûteuse en saison sèche. Au Sénégal, les producteurs s'inscrivent plutôt dans un schéma de diversification de leurs activités. Il s'agit avant tout de sécuriser leur système de production dans un environnement aléatoire. Ils évoluent ainsi vers un système agropastoral plus rassurant, face notamment aux aléas climatiques. Pour eux, il s'agit de diversifier leur revenu tout en minimisant les risques financiers, c'est-à-dire en limitant les charges (achat d'aliments).

A la lumière de leur chiffre d'affaires et dans l'hypothèse que les coûts de production restent modérés (6), ce qui reste à mesurer, il semble que les producteurs mauritaniens trouvent dans leur activité basée sur le lait une ressource importante qui justifie leur relative spécialisation. Les Peuls de Mauritanie (M3) semblent avoir des revenus similaires à ceux d'autres éleveurs, de l'ordre de 1,5 million Fcfa par an, tout en limitant leurs risques d'endettement. En effet, bien que les performances laitières de leurs vaches soient faibles en saison sèche, ces éleveurs font, d'une part, l'économie des dépenses en sous-produits agricoles et, d'autre part, vendent à meilleur prix sur le marché direct en fin de saison sèche (6).

Au Sénégal, le revenu semble être beaucoup plus modeste que sur la rive droite, même si la faiblesse du chiffre d'affaires est compensée par le bas niveau des charges (5). L'activité du lait seule ne semble pas permettre aux éleveurs de faire vivre leur famille. Ils trouvent généralement leur première source de revenu dans la vente de bétail ou, pour certains agro-éleveurs, dans la riziculture (15). Les agro-éleveurs (S3, S4) peuvent toutefois espérer un revenu annuel de 500000 à 1 million de Fcfa (5). Sachant que ce revenu ne comptabilise ni la part autoconsommée par la famille ni la part bue par le veau, cette ressource apparaît donc comme importante, rapportée au niveau de vie au Sénégal. A titre de comparaison, les exploitations agricoles du delta, sur la rive gauche, génèrent un revenu annuel moyen de 900000 Fcfa (13). Par ailleurs, un hectare de riz permet de dégager en moyenne un revenu de 100000 Fcfa à $250000 \mathrm{Fcfa}$ (autoconsommation comprise) alors que la surface irriguée moyenne exploitée par an est inférieure à quatre hectares dans le delta pour 75 p. 100 des exploitants (13). Le revenu laitier serait donc tout à fait comparable au revenu de la riziculture dans les exploitations enquêtées.

Par ailleurs, le lait participe notablement à l'apport protéique dans l'alimentation des familles des producteurs laitiers, si l'on considère la part importante prélevée pour l'autoconsommation. Lorsqu'il est vendu, il permet d'obtenir un revenu régulier et quotidien facilement mobilisable pour les dépenses domestiques, ce qui est exclu pour le riz et les principales cultures de diversification (tomate, oignon). Enfin, les risques d'endettement ou les difficultés d'accès et de remboursement du crédit, problèmes récurrents en agriculture irriguée, sont minimisés par la faiblesse des charges, à l'exception des dépenses en sous-produits agricoles consenties en saison sèche chez les producteurs mauritaniens. Dans ce cas, leur débouché et leur rémunération sont toutefois assurés via les usines de collecte de lait.

\section{Quel avenir pour la production laitière dans le delta? Rôle des structures de collecte}

\section{Rappel historique sur la collecte de lait dans le delta}

Dans un contexte national de déficit chronique en produits laitiers, plusieurs essais de collecte de lait frais ont déjà été menés dans le delta du fleuve Sénégal. Ainsi, sur la rive gauche, une laiterie de l'Union des coopératives laitières (Ucolait) a été installée à SaintLouis au début des années 70. Mais l'expérience n'a duré que quelques années par manque de rigueur dans la collecte (régularité, qualité hygiénique et sanitaire du lait) malgré une volonté affichée des éleveurs de fournir du lait à l'usine (4). Soumise à la concurrence simultanée de produits importés et du commerce informel local, elle n'a pas pu se pérenniser, à l'image des tentatives d'industrialisation laitière menées au Mali et au Niger à cette époque (11).

Sur la rive droite, avec la mise en place de l'usine de collecte de la LM en 1989, les éleveurs issus de toutes les couches sociales se sont orientés vers la commercialisation du lait autour de laquelle s'est organisée la gestion du troupeau. Aujourd'hui, quelques 300 fournisseurs livrent quotidiennement entre 8000 et 11000 litres de lait à l'usine de collecte de la LM, dont les trois quarts en lait de vache (1).

En outre, la dévaluation du franc de la Communauté financière africaine en 1994 a rendu au moins provisoirement la production locale plus compétitive face aux importations de lait en poudre. De plus, le delta offre des atouts indéniables pour son développement : sa tradition pastorale et ses potentialités fourragères. Ainsi, une seconde usine de collecte, Toplait, s'est installée à Rosso-Mauritanie en janvier 1999. Initialement implantée à l'est du pays, cette usine a en effet été confrontée à des problèmes d'approvisionnement en lait frais en saison sèche. Or, contrairement à d'autres régions limitrophes, le delta offre la possibilité de produire toute l'année grâce aux ressources issues de l'agriculture irriguée.

L'avenir de la production laitière dans le delta du fleuve Sénégal apparaît donc relativement prometteur, dans un contexte où les politiques de développement des Etats souhaitent transformer cette zone en un vaste bassin laitier. Pourtant des interrogations subsistent de part et d'autre du fleuve. 


\section{Perspectives prometteuses sur la rive gauche}

Dans la partie sénégalaise du delta, il apparaît que la filière du lait reste informelle en l'absence de structures de collecte bien organisées alors même que le potentiel des ressources alimentaires pour les vaches laitières de la rive gauche est sensiblement supérieur à celui de la rive droite (6). Si le potentiel génétique y est plus faible, les programmes ambitieux d'insémination artificielle engagés depuis quatre années dans la région de Saint-Louis devraient permettre de combler ce retard.

Aussi, il reste une grande marge de progression pour le développement de la production laitière. Ce développement pourrait avant tout s'appuyer sur les agropasteurs Peuls (S3 et S4). Ces éleveurs présentent en effet de nombreux atouts : ils vendent déjà régulièrement du lait, ils se sont sédentarisés autour de leurs champs de riz, ils sont généralement proches de l'axe routier Saint-Louis Richard Toll et ils ont facilement accès à des ressources alimentaires en saison sèche (parcours postculturaux, son de riz).

C'est d'ailleurs sur ce potentiel que souhaite s'appuyer le projet Delta Lait en installant une usine de collecte à Ross-Béthio (4). Pourtant, dans ce schéma de développement, à l'instar des pasteurs mauritaniens, les éleveurs du diéri sont probablement à prendre en compte. Leur potentiel de production est en effet conséquent, à l'image de la taille de leurs troupeaux. Une attention particulière mérite de leur être accordée ainsi que des mesures spécifiques en termes de postes de collecte du lait et de stockage des sous-produits agricoles.

Plusieurs questions restent cependant en suspens. La première concerne la capacité des éleveurs de la rive gauche à produire et à s'organiser dans le sillage de la mise en place de structures de collecte, industrielles ou artisanales. Les expériences privées menées à Rosso en Mauritanie (1) et à Niono au Mali (15) dans des conditions similaires (systèmes irrigués, élevage originellement extensif) incitent à l'optimisme, mais il faut tenir compte des différences de mentalité. Il serait surprenant de voir délaissé un système agropastoral stabilisé et rassurant au profit d'une production spécialisée. Par ailleurs, les femmes sont traditionnellement chargées de la commercialisation du lait. Contrairement à l'expérience mauritanienne, il semble souhaitable pour l'équilibre social au sein des familles qu'elles gardent un rôle central dans la collecte et la rémunération du lait (4). Mais les Peuls, et les femmes en particulier, ont déjà montré qu'ils pouvaient rapidement s'adapter à des bouleversements conséquents dans le delta du fleuve (15).

\section{Poursuite d'un développement rapide sur la rive droite}

Malgré un potentiel fourrager inférieur à celui de la rive gauche, la filière laitière est plus développée sur la rive droite. Un meilleur potentiel génétique du troupeau, une tradition de commerçants et de consommateurs de lait cru chez les Maures expliquent partiellement cette différence. Il semble toutefois que c'est l'implantation de l'usine de collecte de la LM qui a créé l'engouement que l'on connait, avec l'ouverture aujourd'hui de deux usines supplémentaires, l'une à Rosso, l'autre en projet à Bogué.

Le marché visé est celui du lait frais à Nouakchott, à l'image de celui de Dakar pour le projet proposé sur la rive gauche. La collecte est faite dans le delta, dans un rayon de $50 \mathrm{~km}$ autour de l'usine, et le lait frais réfrigéré est envoyé par camion-citerne à la capitale où il est conditionné et transformé. Aussi, contrairement à ce qui est traditionnellement décrit pour les marchés ouest-africains $(10,16)$, il ne s'agit pas ici d'une production périurbaine, mais plutôt d'un bassin de production situé à 200-250 km du centre de consommation pour un produit hautement périssable en milieu tropical. Il est essentiel de contrôler régulièrement la collecte, le transport et la qualité hygiénique et sanitaire du lait pour pérenniser ce système ; la confiance semble devenir le maitre mot entre les différents acteurs. C'est le pari réussi par la filière du lait dans le delta du fleuve Sénégal, sur la rive droite.

Par ailleurs, la dépréciation de l'ouguiya en Mauritanie ces deux dernières années face au franc de la Communauté financière africaine (monnaie sénégalaise) de l'ordre de 20 p. 100 a sensiblement augmenté le coût de revient du litre de lait en Mauritanie, dans la mesure où la production dépend beaucoup des sous-produits importés du Sénégal. Il faut donc chercher des solutions pour réduire les coûts de l'éleveur afin de lui permettre de dégager un meilleur revenu de son activité de production laitière et limiter les risques d'un investissement non rentable. Trois principaux axes peuvent y contribuer techniquement : le développement des cultures fourragères (à privilégier chez les agro-éleveurs du waalo), un rationnement raisonné (quantité et qualité, rations individualisées) des vaches laitières et le suivi sanitaire des animaux notamment dans la zone de Keur Macène (ouest de Rosso).

Compte tenu de son prix concurrentiel et des habitudes alimentaires mauritaniennes, le lait frais semble encore avoir de solides arguments pour résister aux marchés du lait en poudre importé et du lait caillé. Aujourd'hui, les éleveurs semblent privilégier la vente à l'usine de collecte ; le nombre croissant des fournisseurs en est la preuve. Ils y trouvent notamment une sécurité de vente. Mais, avec la mise en place d'une seconde usine de collecte début 1999 (Toplait), le paysage économique risque de se modifier. On peut tout d'abord noter que ce nouveau contexte concurrentiel a joué en faveur des éleveurs par l'augmentation du prix d'achat de leur lait, faute d'offre ou par excès de demande. C'est ainsi que la LM a augmenté son prix d'achat de 90 à $110 \mathrm{UM} / \mathrm{l}$. A moyen terme, il est plus difficile de pronostiquer un avenir serein pour les éleveurs et pour la filière du lait dans son ensemble. Que deviendra la concurrence entre les deux usines privées sur un marché relativement petit et dans un secteur où les marges sont faibles ? Les éleveurs serontils capables d'augmenter rentablement leur production ? La mise en place d'une seconde usine de collecte n'encouragera-t-elle pas l'arrivée de nouveaux troupeaux sur un espace pastoral déjà réduit? Quels types d'éleveurs faudra-t-il prioritairement privilégier ou aider? Autant de questions sur lesquelles l'ensemble des acteurs de la filière devront rester vigilants afin d'en garder la maîtrise.

\section{- CONCLUSION}

A l'échelle de l'exploitation ou de la région, cette étude montre qu'aujourd'hui la production laitière peut être considérée comme une réelle activité de diversification dans les systèmes irrigués du delta du fleuve Sénégal. En outre, même s'ils ne sont pas riziculteurs, les producteurs de lait ont maintenant des pratiques d'alimentation plus intensives et sédentaires que par le passé, qui dépendent des ressources issues de la culture irriguée.

Aussi, en intégrant l'agriculture et l'élevage dans le delta, la production laitière apparaît comme une véritable activité de diversification économiquement importante sur la rive droite et en passe de le devenir sur la rive gauche. Face à l'augmentation des effectifs et à la réduction de l'espace pastoral, des études ultérieures visant à un partage négocié des ressources entre utilisateurs devront être menées. Des études devront également être menées afin de préciser le revenu issu de la production laitière en fonction des différents types d'éleveurs identifiés. Ces revenus pourront alors être comparés à ceux du riz et des autres productions de diversification (cultures et viande).

La présente étude renvoie une image bien différente de celle, maintes fois exposée, d'un élevage contemplatif à proscrire absolument des casiers rizicoles parce que non productif et facteur de 
dégradations et de conflits. Les populations concernées directement par les revenus du lait représentent d'ailleurs une part non négligeable de la population rurale du delta (entre 30 et 50 p. 100 ; $12,13)$, ce qui permet de mieux mesurer encore l'importance réelle de cette activité dans cette zone. A l'instar des trois dernières décennies dévolues invariablement à l'essor de la riziculture, les décideurs locaux et nationaux auront désormais à prendre en compte cette dimension socio-économique conséquente pour décider, en toute connaissance de cause, de l'avenir de la production laitière au sein des systèmes irrigués.

\section{BIBLIOGRAPHIE}

1. ABEIDERRKHMANE N., 2001. Collecte, transformation et commercialisation du lait pour I'approvisionnement de Nouakchott I'expérience de la Laitière de Mauritanie. In : Duteurtre G. ed., Atelier de restitution d'ATP " lait ", septembre 1998. Montpellier, France, Cirademvt (sous presse).

2. AUDRU J., 1966. Etude des pâturages naturels et des problèmes pastoraux dans le delta du fleuve Sénégal ; tome 1 : description du milieu ; tome 2 : politique de l'élevage. Maisons-Alfort, France, lemvt, 359 p.

3. BOUDET G., 1989. Connaissance et gestion de I'espace pastoral sahélien. In Audru et coll. eds, Terroirs pastoraux et agropastoraux en zone tropicale. Maisons-Alfort, France, Cirad-lemvt, p. 1-59. (Etudes et synthèses $\left.n^{\circ} 24\right)$

4. CACG, 1995. Projet Delta-Lait. Etude de faisabilité du développement de la production laitière dans le delta du fleuve Sénégal. Rapport d'étude. Saint-Louis, Sénégal, Caisse française de développement, Saed, 139 p.

5. CORNIAUX C., LE MERCIER J., SALEM M., DIA A.T., YADDE A., 2000. Production de lait de vache en rives droite et gauche du delta du fleuve Sénégal : une réelle activité de diversification en systèmes irrigués ? In : Legoupil J.C. ed., Pour un développement durable de I'agriculture irriguée dans la zone soudano-sahélienne, Actes du séminaire Pôle systèmes irrigués, Dakar, Sénégal, 30 nov. - 03 déc. 1999. Paris, France, Psi/Wecard - Coraf, p. 140-159.

6. CORNIAUX C., SALEM M., DIA A.T., 2001. Production et commercialisation de lait de vache en systèmes irrigués : cas de la rive droite du delta du fleuve Sénégal (Mauritanie). In : Actes du séminaire Alimentation, santé et productivité des ruminants dans les systèmes

\section{Summary}

Corniaux Ch., Le Mercier J., Dia A.T. Cow Milk Production in the Delta of the Senegal River: Real Farming Diversification within an Irrigated System?

Development of irrigated farming on a large scale dramatically changed the environment of the Senegal River delta. Regardless of the changes, milk production increased on the right bank (Mauritania) and remained stable on the left bank (Senegal). Studies were carried out from July 1997 to June 1999 to better assess interactions between animal husbandry and irrigated fields. Results are presented on the use of forage resources and on some technical/economical parameters. Milk production at the farm level appeared as a truly profitable activity of diversification for the relatively large population of cattle breeders/rice farmers. On the right bank, it appeared rather as a specialized field. Its future is promising as the area is on its way to becoming a milk production basin that will supply the capital cities of Nouakchott and Dakar. The central role of milk collection is highlighted.

Key words: Cow milk - Diversification - Profitability - Rice field - Irrigated farming - Senegal - Mauritania. d'élevage ruraux d'Afrique sahélienne et soudanienne, Dakar, Sénégal, 24-27 octobre 2000 (sous presse).

7. ENDA, 1986. Engelhard et Ben Abdallah Eds, Enjeux de I'aprèsbarrage : vallée du Sénégal. Paris, France, La documentation française, $632 \mathrm{p}$.

8. HUAT J., DAVID-BENZ H., 2000. La tomate d'industrie au Sénégal : performances de la production et enjeux pour la filière. In : Legoupil J.C. ed., Pour un développement durable de l'agriculture irriguée dans la zone soudano-sahélienne, Actes du séminaire Pôle systèmes irrigués, Dakar, Sénégal, 30 nov. - 03 déc. 1999. Paris, France, Psi/Wecard - Coraf, p. 167-187.

9. JAMIN J.Y., TOURRAND J.F., 1986. Evolution de I'agriculture et de I'élevage dans une zone de grands aménagements. Cah. Rech. Dév., 12 : 595-609.

10. LE MASSON A., 1996. Programme national de promotion de la production laitière au Mali - Les systèmes d'élevage à vocation laitière : contraintes, propositions. Montpellier, France, Cirad-emvt, 95 p. (Rapport)

11. LESSOR 1993. Le lait au Sahel : marchés locaux et marché international. Rennes, France, Cereteb, p. 333-343. (Compte rendu de fin d'études)

12. ONS, 1998. Annuaire statistique de la Mauritanie. Année 1996. Nouakchott, Mauritanie, Office national de la statistique, Service de la conjoncture, 149 p.

13. SAED, 1997. Recueil des statistiques de la vallée du fleuve Sénégal. Annuaire 1995/1996 - version détaillée. Saint-Louis, Sénégal, Saed, 142 p.

14. SANTOIR C., 1993. Des pasteurs sur les périmètres. In : Boivin P., Dia I. et coll. eds, Nianga, laboratoire de l'agriculture irriguée en moyenne vallee du Sénégal, Actes de I'atelier Orstom/Isra, Saint-Louis, Sénégal, 19-21 octobre 1993, p. 375-405.

15. TOURRAND J.F. 1993. L'élevage dans la révolution agricole du waalo : ruptures et continuité. Thèse d'Etat, Université de Paris XII, Créteil, France, $415 \mathrm{p}$.

16. VATIN F., 2001. Le lait et la ville : Paris-Dakar - Perspective historique et philosophie du développement. In : Duteurtre G. ed., Atelier de restitution d'ATP " lait », septembre 1998. Montpellier, France, Cirademvt.

Reçu le 13.06.2000, accepté le 07.11.200

\section{Resumen}

Corniaux Ch., Le Mercier J., Dia A.T. Producción de leche de vaca en el delta del río Senegal: una actividad real de diversificación en sistemas irrigados?

A pesar de un contexto profundamente modificado por la realización a gran escala de cultivos irrigados, la producción láctea se ha desarrollado sobre la margen derecha (Mauritania) y se ha mantenido sobre la margen izquierda (Senegal) del delta del río Senegal. Los trabajos, llevados a cabo entre julio de 1997 y junio de 1999, permitieron una mejor evaluación del grado de interacción entre la cría y la agricultura. Se presentan los resultados sobre la utilización de los recursos forrajeros y sobre ciertos parámetros técnico económicos. La producción de leche aparece como una verdadera actividad rentable de diversificación, a escala de explotación, para los agro pastores, relativamente numerosos sobre la margen izquierda. Sobre la margen derecha, parece corresponder más a una especialización. El futuro de esta actividad parece prometedor en esta zona destinada a convertirse en un lecho de producción láctea para el abastecimiento de las capitales Nouakchott y Dakar. Se subraya el papel central de la colecta.

Palabras clave: Leche de vaca - Diversificación - Rentabilidad - Arrozal - Agricultura de regadio - Senegal - Mauritania. 